\title{
Pode-se aprender OpenMusic em três dias?
}

\author{
Mikhail Malt \\ Ircam - MINT - Paris IV \\ Mikhail.malt@ircam.fr \\ Tradução ': Yuri Behr \\ USP \\ yuribaer@gmail.com
}




\section{Resumo}

Como organizar o ensino da composição assistida pelo computador? Iremos expor algumas sugestões e questões provenientes da nossa experiência na área da pedagogia de meios informáticos destinados a jovens compositores. Depois de ter exposto o contexto do ensino e do público-alvo, faremos uma distinção entre "informática musical"1 e "música informática". serão analisados os diversos pontos que nos parecem importantes a serem ensinados/ou aprendidos. Entre eles, serão discutidos: os fundamentos, as ferramentas, a formalização, as ferramentas de formalização e de cálculo, a relação entre estruturas formais e a sonificação, a história das técnicas de EAC (escrita assistida por computador) e a integração com a prática. Essa reflexão, ainda que focada sobre a EAC, procura ser a mais abrangente possivel e aplicável a outras disciplinas musicais, usando tecnologias digitais ou outras.

Este texto é dedicado a João Dias Carrasqueirra e Josette S. de Melo Feres, meus dois modelos pedagógicos e musicais. 


\section{Introdução}

Os novos alunos frequentemente perguntavam ao "mestre ${ }^{1}$ de música" brasileiro João Dias Carrasqueirra quanto tempo demoraria para aprenderem a tocar a flauta. Com seu olhar compassivo, mas também provocador e brincalhão, ele invariavelmente respondia: "Não tenho a mínima ideia, eu mesmo ainda não terminei".

Este texto é uma reflexão pessoal sobre o ensino de Composição Assistida por Computador (CAC) (ou, como preferimos denominá-la, Escrita Assistida por Computador - EAC2), que foi realizado há mais de ${ }^{3}$ vinte anos, dentro do contexto do "Ciclo de composição e de informática musical" ${ }^{4}$ no IRCAM. Este ensino é dirigido principalmente a jovens compositores experientes, dentro de uma tradição de escrita musical clássica.

Nós não pensamos, como o título sugere, que o uso da EAC esteja reduzido ao ambiente OpenMusic (AGON, 1998). Um grande número de compositores ${ }^{5}$ usa com sucesso outros ambientes de computador para a tarefa de assistência à escrita musical. Esperamos, no entanto, que as reflexões que seguem sejam suficientemente gerais para que possam beneficiar a todos.

\section{0 contexto do ensino ${ }^{6}$}

Por volta de 1970, um novo termo especializado aparece na língua francesa:

"informatique musicale". Seu uso não se tornou geral até a década de oitenta, mas as primeiras aparições desta expressão constituem um fato notável. Da "Música calculada" - como se dizia na França no início da década de 1960 - até a "informatique musicale", a diferença de significado não é uma simples nuance. Obviamente, há uma problemática entre música e computadores, mas o ponto em questão é se uma transformação significativa está associada ao surgimento e ao uso desse termo. (VEITL, 1983, p. 1).

N. do A.: O professor João Dias Carrasqueira não era só um professor de música, mas um verdadeiro "Mestre de Música" à imagem dos "Maîtres de musique" que acolhiam estudantes para viverem com eles, transmitindo-Ihes a música, não dentro do contexto de um tempo de aula previamente estipulado, mas dentro de uma vivência musical.

2 Para a diferença entre CAC e EAC, veja Malt (2009, p. 178-183).

3 N. do T.: O original deste texto é de 2012.

$4 \quad$ N. do T.: O original deste texto é de 2012.

$5 \quad$ N. do A.: Título original: "Cursus de composition et d'informatique musicale". Este ensino continua ativo e se desenvolvendo, adaptando-se às mudanças de contexto, sociais, musicais e tecnológicas. Atualmente, há uma reflexão sobre a evolução deste ensino, não somente para compositores de música dita "clássica" ou contemporânea, mas também para todos os criadores artísticos usando o som.

$6 \quad$ N. do A.: Esta seção é destinada principalmente ao leitor de origem francesa. Como é explicado no texto, houve um erro (ou uma escoIha) na tradução do termo computer music (música feita por computadores etc.), que, na opinião do autor, teve uma influência nefasta na evolução desta área na França. Há uma história de quando Jean-Claude Risset assistiu à apresentação deste texto nas Journées d'informatique Musicale em 2011. Nessa ocasião, Jean-Claude Risset, que estava ao meu lado na hora do almoço, virou-se para mim e disse: "Mikhail, je pense que c'est ma faute" (Mikhail, acho que isso é culpa minha!), justificando que, aparentemente, na pressa de achar uma tradução adequada ao termo "computer music" para um congresso internacional, ele teria optado por "informatique musicale" em vez de "musique informatique" ou "musique par ordinateur", pois "informatique musicale" era mais elegante, mais "sonante". Mas rapidamente ele concordou que esta tradução era problemática. 
O ensino da CAC na França está situado no contexto do que se convencionou chamar de "computação musical" (informatique musicale). O problema com essa denominação está no fato de que ela induz a um deslocamento de significado em relação à "Computer Music". Quando usamos o referido termo anglo-saxão7, estamos falando de música produzida com o computador, desde o uso de computadores até uma realização musical (interpretação, composição e estudo posterior) (ROADS, 1994, p. ix). Em um breve paralelo com a análise gramatical, podemos dizer que a "music" é o substantivo - neste caso, o foco da expressão -, e "computer" é o predicado, o epíteto que designa uma propriedade do substantivo. "Nós" fazemos música com a ajuda da computação. Em francês, essa expressão foi traduzida como "informatique musicale". Esta tradução é ambígua e equivocada. Em nosso caso particular, o deslocamento do significado não durou muito; "informatique" é agora o substantivo, o foco de expressão, e a propriedade "musicale", o predicado. "Nós" fazemos, então, informática, computação, aplicada à música! A música é aqui um dos campos de aplicação de uma disciplina principal: a informática, computação. É por esta razão que, no contexto do nosso trabalho, gostaríamos de propor o termo "música informática"8, ou "música computadorizada". A informática musical (ou computação musical) trata da pesquisa e do desenvolvimento de ferramentas para estudo, interpretação e composição musical; enquanto a "música informática" (ou música computadorizada) é o uso da informática para estudar, interpretar e compor música. Embora essa diferença possa parecer academicamente pedante, é de extrema importância na definição de metas em um quadro pedagógico e de pesquisas. Para fazer uma metáfora, a computação musical é o estudo de luthieria (em tudo que há de mais nobre), enquanto a música computadorizada é a arte de interpretação e de uso de ferramentas (e, de agora em diante, das tecnologias em geral) aplicadas à música (estudo, interpretação e composição). Obviamente, essas duas disciplinas não possuem fronteiras estritas ${ }^{9}$. O estudo da música informática exigirá frequentemente um percurso nos campos da informática musical. Assim como um oboísta (ou um fagotista) deve dedicar-se à confecção de palhetas, seu objetivo é claro, ele é um intérprete, não um luthier.

O campo de estudo e aplicação da EAC é definido em dois espaços: a criação de ferramentas e o uso de ferramentas. Como resultado desta reflexão, tentaremos apresentar a experiência de ensino e aprendizagem da EAC em um quadro bastante preciso de música informática (uso de ferramentas), ou música e tecnologia, para um público de jovens compositores.

\footnotetext{
7 Consideraremos a aplicação do termo música informática desde a década de 1960 até a década de 1980. A partir da década de 1990, também designará uma corrente estética de música eletroacústica.

8 Outras traduções também podem ser adaptadas. "Música computacional" (neologismo anglo-saxão), "música por computador" etc. Em português, "música computadorizada". Atualmente, esta denominação está sendo também substituída por "Música e tecnologia". N. do A.: Gostaríamos de lembrar que a "Computer Music" é definida como a intersecção das áreas de Música, Ciências e Tecnologias.
}

9 N. do A.: Gostaríamos de lembrar que a "Computer Music" é definida como a intersecção das áreas de Música, Ciências e Tecnologias. 


\title{
30 que é a CAC?
}

Como já escrevemos em outra ocasião:

\begin{abstract}
O auxílio à composição ou mais precisamente a escrita assistida por computador (EAC) é uma disciplina bastante recente que nasceu nos anos 50 e 60, principalmente sob os experimentos pioneiros de Hiller (Illiac Suite, 1956) e Xenakis (desde a formalização da Achorripsis, 1956-57, até o "ST", 1962, que foram calculados por computador). O seu desenvolvimento está intimamente ligado à evolução da composição musical automática $(\mathrm{CMA})^{10}$, ou à formalização algorítmica e musical e, obviamente, à evolução das plataformas de hardware e software. (MALT, 2009, p. 163).
\end{abstract}

Em nosso caso específico, consideraremos o termo EAC como "qualquer processo informático que possa acelerar o trabalho do compositor em seu processo criativo" (MALT, 2009, p. 183). Porquanto a EAC seja "a determinação das posições dos objetos em um espaço musical predefinido pelo compositor", o espaço musical é "Cada conjunto de parâmetros que representam uma realidade musical e/ou sonora" (MALT, 2009, p. 203). Esta disciplina evoluiu consideravelmente, tornando-se um campo independente de estudo e prática. Ela é atualmente ensinada em várias instituições de prestígio na Europa, América e Ásia (IRCAM, CNSMDP, Departamento de Música de St. Etienne, Universidade Columbia, Universidade de Harvard, CNMAT - Berkeley, Universidade de Montreal, Hanyang Universiy etc. ${ }^{11}$ e praticada por um número cada vez maior de compositores ${ }^{12}$.

\section{0 ensino da EAC}

Como ensinar EAC? Perguntar o que ensinar é, muitas vezes, saber o que se precisa aprender. Então, o que precisamos saber para aprender a dominar a EAC? Como evolui o aprendizado da EAC?

Após alguns anos de experiência docente, gostaríamos de propor um aprendizado de EAC dividido em sete partes ${ }^{13}$ : os pré-requisitos, a ferramenta, a modelização e formalização, as ferramentas de formalização e cálculo, a relação entre estruturas formais e sonificação, a história das técnicas de EAC e a integração.

\footnotetext{
10 N. do T.: Também conhecida por música algorítmica ou, como é recentemente denominada, música generativa.

11 N. do T.: Atualmente, a composição assistida por computador ou escrita musical assistida por computador é também ensinada em várias instituições brasileiras, como, por exemplo, Unicamp, USP, UFRJ, UFB, UFPB, dentre outras.

12 Vide: Agon, Andreatta, Assayag, Schaub (2004); Agon, Andreatta, Bresson (2006); Hirs e Gilmore (2009).

13 N. do A.: Evidentemente, "sete partes" não é um dogma esotérico, mas uma classificação ad-hoc sujeita a ser adaptada e corrigida quando for conveniente.
} 


\subsection{Os pré-requisitos}

Um pré-requisito fundamental para o estudo da EAC é o conhecimento e a prática da escrita musical (composição) e das disciplinas relacionadas (harmonia, contraponto, orquestração, escrita etc.). Não confio no uso de algoritmos, de caixas-pretas, por compositores que não dominam uma forma de escrita musical. A EAC é uma assistência à composição e à escrita, e não um substituto para a falta de domínio ou de conhecimento. No entanto, isso não deve ser confundido com o uso da EAC como um laboratório para a exploração da escrita musical ${ }^{14}$.

\subsection{A ferramenta}

A ferramenta é o software-base em que a EAC pode ser desenvolvida. Existem hoje em dia muitos ambientes de computação que podem ser usados para a EAC, tais como: OpenMusic (@ IRCAM) ${ }^{15}$, PWGL (@) Mikael Laurson) ${ }^{16}$, Max/MSP (@ Cycling '74) ${ }^{17}$, PureData (por Miller Puckette) ${ }^{18}$, Elody (C GRAME) ${ }^{19}$, Common-Music (@ Rick Taube) ${ }^{20}$ etc ${ }^{21}{ }^{22}$. O que importa é ressaltar que cada ferramenta, cada ambiente, oferece uma representação ou paradigma de cálculo específico. Cada paradigma de representação induz a um espaço operacional, condicionando o uso do ambiente computacional ${ }^{23}$.

Um ambiente de desenho gráfico (como o Xenakis UPIC, por exemplo) induzirá seu usuário a manipulações geométricas, deslocamentos, operações de escala etc. em seus objetos musicais. No entanto, ambientes de programação gráfica como OpenMusic ou Max/MSP induzem o usuário a criar processos baseados em cálculo.

O tempo de aprendizagem da "sintaxe", da "lógica" e da totalidade ou grande parte dos elementos que constituem um ambiente é variável, dependendo da habilidade e da capacidade do compositor, bem como das diferentes possibilidades oferecidas pelo próprio ambiente. Esta aprendizagem, que pode ser alcançada a médio prazo, é muitas vezes uma questão de trabalho constante e contínuo.

\footnotetext{
$14 \quad$ N. do A.: Gostaria de lembrar ao leitor o comentário de Pierre Boulez a respeito da inteiração do compositor com a máquina, o computador. Pierre Boulez nos diz que o que o interessava era o fato de que a máquina às vezes respondia a perguntas que o compositor não havia feito (BOULEZ; GREUSSAY, 1988, p. 129-137).

15 Disponível em: <http://repmus.ircam.fr/openmusic/home>.

16 Disponível em: <http://www2.siba.fi/PWGL/>

17 Disponível em: <www.cycling74.com/>.

18 Disponível em: <http://crca.ucsd.edu/ msp/software.html et http://puredata.info/>

19 Disponivel em: <http://www.grame.fr/Recherche/Elody/>

20 Disponível em: <http://commonmusic.sourceforge.net/>.

21 Uma lista não exaustiva de ambientes dedicados, ou que pode ser usada para CAC, pode ser encontrada em <http://www.flexatone.net/ algoNet/sysTitle.html>.

22 N. do A./T.: É conveniente destacar que, assim como as demais plataformas de computação, os ambientes de EAC estão em constante desenvolvimento. Nesse sentido, é pertinente destacar que, muito embora o OpenMusic continue sendo a principal plataforma, outras surgiram, com destaque para o Opusmodus (https://opusmodus.com). Com o desenvolvimento da Biblioteca Bach (http://www.bachproject.net), também é possivel implementar um tipo de EAC em MAX/MSP (๔ Cycling '74).

Por outro lado, outras tendem a desaparecer, como o PWGL, que não é mais subsidiado pela Universidade de Helsinki. 
Este ponto frequentemente requer uma reflexão, mesmo que a aprendizagem de uma ferramenta seja a base fundamental, material e computacional para a prática da EAC, não é o único elemento. Um erro habitual é reduzir o aprendizado da disciplina ao domínio da ferramenta. Este é um mal-entendido bastante difundido ${ }^{24}$. $O$ mesmo ocorre com as outras disciplinas da música informática, como o tempo real, análise e síntese, criação eletroacústica etc. É importante não confundir ferramentas e disciplinas. $O$ restante deste texto esclarecerá este ponto.

\subsection{A modelização e a formalização}

Um dos principais problemas da EAC, e da ciência da computação em geral, diz respeito à diferença entre a linguagem que o homem usa para se expressar (LEVY, 1987, p. 31) e as linguagens formais que regem o comportamento dos computadores. O primeiro passo para poder usar os ambientes de EAC é poder modelizar o problema musical (ou uma parte) e formalizá-lo, propondo um algoritmo de cálculo. Como já comentamos: "[...] com a EAC, a formalização tornou-se uma necessidade, uma ferramenta, um imperativo para comunicar-se com a máquina" (MALT, 2009, p. 214).

Infelizmente, a formalização está longe de ser uma prática difundida entre os compositores ou entre os músicos. A grande maioria está acostumada a trabalhar com a intuição e a imaginação. A chegada da máquina e dos ambientes informáticos abalou essas práticas. Aprender modelização e formalização é um passo essencial para uso e controle da EAC. O problema não é apenas modelizar estruturas musicais, o que já exige um grande esforço, mas também modelizar o "próprio pensamento", fazendo certo número de perguntas, tais como: "Quais representações devo usar?", "Quais são as representações mais efetivas para representar os conceitos que eu manipulo?", "Qual é a eficiência 'operativa' desses conceitos?". ${ }^{25}$

É importante, no âmbito da EAC, usar representações operacionais ou aquelas que sejam coerentes com o contexto do ambiente utilizado. ${ }^{26}$

A modelização e a formalização são disciplinas bem conhecidas pelas ciências, notadamente a física, que há muito questiona suas consequências epistemológicas. $\mathrm{Na}$ música, modelização e formalização são disciplinas mais recentes que precisam ser desenvolvidas. A partir deste ponto (e para os seguintes: 4.4, 4.5 e 4.6), será muito útil usar o "estudo de caso"27 como estratégia de ensino, ou seja, usando a EAC.

\footnotetext{
24 N. do A.: O mesmo problema existe, por exemplo, em astronomia ou computação.

25 N. do A.: Para entender o que é "eficiência" operativa, basta pensar que precisamos fazer uma divisão. Que representação numérica vamos usar? Números arábicos ou números romanos? É fácil entender que, neste caso, a representação numérica arábica permite o desenvolvimento de algoritmos de cálculo mais simples e eficientes.

26 N. do A./T.: Gostaríamos de relembrar que existem modelos formais que se expressam de forma que possam ser calculados; e modelos não formais, que não implicam diretamente um algoritmo de cálculo. O que é chamado de "modelo operacional", neste texto, são os modelos formais, os que podem dar origem a um algoritmo.
}

27 N. do A./T.: O que chamamos "estudo de caso" é o estudo da formalização em obras do repertório musical. 


\subsection{Ferramentas de formalização e cálculo}

Para poder estabelecer modelos formais, também é necessário ter uma caixa de ferramentas operacionais, conhecer tanto conceitos matemáticos como estruturas de cálculo algorítmicas. Tomemos um problema elementar ${ }^{28}$ : construir um acelerando com base numa duração de partida, uma duração de chegada, um número de passos e um fator multiplicativo. A solução desse problema é bem mais fácil se reconhecermos no enunciado uma "série geométrica". A atitude do compositor, no uso da EAC, dentro de um quadro de criação, é bem mais semelhante à do engenheiro do que a do cientista. O compositor tenta usar ferramentas conceituais e/ou de software para resolver problemas musicais e de formalização. Não procura desenvolver teorias científicas ou novas técnicas algorítmicas, exceto, obviamente, em períodos de pesquisa musical. Mesmo neste contexto, sua pesquisa é uma busca por "meios de criação". No entanto, esse é outro assunto. Adquirir esse conhecimento é um pré-requisito para as etapas de modelagem e formalização. ${ }^{29}$

\subsection{A aprendizagem das relações entre estruturas formais e sua sonificação}

Conhecer as ferramentas e algoritmos de cálculo ainda não é o suficiente. Também é necessário estabelecer relações entre o resultado do cálculo e a sua sonificação, ou seja, a conversão de dados numéricos em um parâmetro do espaço musical (alturas, durações, tempo, timbre etc.). Qual é o resultado musical da geração de uma nuvem de alturas com uma distribuição gaussiana, ou Arcsinus ${ }^{30}$ ? Quais são as ferramentas conceituais para formalizar características musicais específicas? O compositor precisará adquirir outro conhecimento.

O compositor que quer aproveitar as novas possibilidades oferecidas pela ciência da computação deve ir além do "como fazer"31 e chegar ao "saber como fazer". Ele terá que desenvolver um novo solfejo que chamaremos: "Um solfejo de modelos". (MALT, 2009, p. 215).

Se entendermos o solfejo musical clássico como a capacidade de vincular o com-

\footnotetext{
28 N. do A.: Este exemplo faz parte de um problema mais geral que nos foi proposto pelo compositor Luis Fernando Rizo-Salom, durante o Cursus no IRCAM em 2005.

29 N. do A./T.: Um nível de conhecimento de matemática correspondente ao primeiro ano do curso universitário de Matemática seria o mínimo, o que equivale, em linhas gerais, às seguintes disciplinas: Introdução à Computação, Introdução à Probabilidade e à Estatística I, Cálculo Diferencial e Integral I, Vetores e Geometria, Princípios de Desenvolvimento de Algoritmos, Cálculo Diferencial e Integral II, Álgebra Linear I. Neste ponto, é também útil ter conhecimentos básicos de algorítmica.

30 Sobre o uso da distribuição de Arcsinus para geração de alturas na modelagem computador de Herma de Xenakis, veja, por exemplo: Agon, Andreatta, Assayag, Schaub (2004).

$31 \quad$ N. do A.: Jogo de palavras com a expressão "know-how".
} 
portamento de eventos sonoros (musical) ao comportamento de um espaço gráfico de notação, ou seja, de conectar o senso de um espaço gráfico ao senso de um espaço acústico (ou vice-versa), o que chamamos de "solfejo de modelos" será uma extensão. 0 compositor será capaz de relacionar o comportamento de um modelo de cálculo com os resultados acústicos da sonificação ${ }^{32}$ e, o mais importante, de prever qual modelo de cálculo (ou modelo computacional) representará melhor sua imaginação musical. $O$ compositor será, então, capaz de usar modelos matemáticos levando em consideração suas características, de modo a poder usá-las não como simples algoritmos (BIDLACK, 1992), mas como ferramentas para a formalização de conceitos musicais (LEVY, 1987), ferramentas de escrita musical. Mesmo que pareça uma ficção científica, graças à experimentação e à prática, ainda que seja o loop retroativo "formalização < > simulação", muitos compositores podem hoje prever o comportamento musical dos algoritmos computacionais (MALT, 2009, p. 217-218). Como relatado por Xenakis: "As fórmulas matemáticas são, portanto, domesticadas e subjugadas pelo pensamento musical" (XENAKIS, 1981, p. 47).

A construção de um solfejo de modelos não é a elaboração de um catálogo, mas o desenvolvimento de uma faculdade cognitiva que estabelece relações entre espaços de representações diferentes.

\subsection{História das técnicas da EAC}

Contamos atualmente com mais de meio século de uso do computador em composição musical (música algorítmica e escrita assistida por computador) e o surgimento de estéticas musicais mais ou menos formalizadas que precisam de um cálculo para se reificar (como serialismo ou a música espectral). Além disso, o uso da computação para o cálculo de estruturas musicais gradualmente deu origem a uma série de preocupações comuns ${ }^{33}$, das quais podemos citar a "quantificação" (transição de uma representação contínua no tempo para uma representação discreta e métrica), o uso gráfico de curvas para controle de processo, a sincronização de processos, o uso de crivos para a formalização de alturas e tempo, a interpolação etc. O estudo e a análise das soluções já estabelecidas evitarão a síndrome da "redescoberta da roda", que é muito comum na aprendizagem da música e das tecnologias, especialmente em países que não possuem ensino especializado.

\subsection{A integração dessas conquistas dentro de um contexto de criação}

Chegamos à última parte, e a mais árdua. Primeiro, o compositor deve se questionar sobre a necessidade de usar a EAC. Nós sempre pedimos para que os nossos alunos

\footnotetext{
32 N. do T.: Sonificação refere-se ao uso de dados provenientes de outros estímulos sensoriais, que são convertidos em parâmetros sonoros. Por exemplo, uma imagem em gradientes de cinza pode ser utilizada para parametrizar o timbre através da abertura de um filtro passa alta, ou ainda - de maneira mais simples - para fazer uma gradação entre grave e agudo.

33 Podemos achar algumas dessas preocupações em Agon, Andreatta, Bresson (2006); Agon, Andreatta, Bresson (2009); Hirs e Gilmore (2009).
} 
reflitam sobre isso. O uso da EAC deve ser feito de acordo com uma necessidade musical específica, quer seja porque o compositor deseja e tem uma necessidade de integrar esse novo conhecimento, essa nova metodologia de trabalho em seu próprio processo de composição, quer seja porque ele quer usá-lo em um contexto de experimentação no processo de composição.

O trabalho de integração é longo e requer que o compositor transforme seu pensamento não apenas no processo musical, mas o próprio pensamento em si: o compositor começa um estudo crítico de sua própria reflexão ${ }^{34}$ (MALT, 2009, p. 222).

Esta fase não ocorre em três dias (independentemente das habilidades do compositor), ela é um trabalho de longo prazo. Uma vez que a integração esteja completa, ela permite que o compositor mude sua própria abordagem da composição, fazendo-o descobrir os limites entre o seu ofício (hábitos) e a sua criatividade. O compositor é capaz, então, de pensar a música calculando-a. Quanto às novas gerações de compositores usando a EAC, percebemos que, cada vez mais, o espaço de formalização se funde com o espaço de escrita. Eles têm cada vez menos dificuldade em ligar o cálculo e a intuição, navegando em um espaço de trabalho onde se resvalam o piano, o computador e a folha de papel ${ }^{35}$.

\section{Ensino}

Qualquer ensino de EAC que não leve em consideração estes poucos pontos não pode ser considerado completo. No entanto, percebemos que estabelecer uma formação tão abrangente torna-se difícil e dispendioso quando considerada a relação entre o número de pessoas que possam estar interessadas e as condições necessárias (tempo, espaço e recursos). Porém, mesmo que essas condições materiais (ou outras) nem sempre nos permitam estabelecer um ensino que abarque todos os assuntos expostos, é importante ter em mente o quadro completo. As circunstâncias, sejam elas as restrições comuns do espaço, do tempo e dos recursos materiais, às vezes têm a infortunada tendência de nos fazer esquecer e de nos acomodar, tendo, no nosso caso, uma degradação, a médio ou longo prazo da disciplina em questão.

Ensinar é uma memória de saberes, é uma forma de reatualizar práticas, mantê-las vivas, organizando-as e colocando-as em perspectiva ${ }^{36}$. Para isso, o ensino deve ser ativo. Ele não pode ser uma simples transmissão, a repetição de um discurso congelado. Deve ser um compartilhamento de saberes operante, vibrante, dinâmico, levando à construção de novos conhecimentos e à reorganização dos antigos. Por esta razão, o docente deve, tanto quanto possível, ser um praticante que constantemente se questiona sobre sua própria prática, sobre a sua própria experiência. Isso se aplica à EAC e, sem dúvida, a todas as disciplinas da música computadorizada.

\footnotetext{
$34 \quad$ N. do A.: Uma reflexão sobre o que é formal e o que é informal no seu pensamento. Ou seja, a fronteira entre a sua técnica e a sua criatividade.

35 Marco Stroppa e Hector Parra nos relataram que, no trabalho de composição, eles trabalham assim.

36 Podemos achar algumas dessas preocupações em Agon, Andreatta, Bresson (2006); Agon, Andreatta, Bresson (2009); Hirs e Gilmore (2009).
} 
Em termos de trabalho em sala de aula, e de uma pedagogia destinada aos jovens criadores, a melhor estratégia até agora, em nossa opinião, tem sido a "pedagogia de projeto". Um ritmo de trabalho pontuado por "exercícios de estilo" combinando um objetivo técnico de compreensão do ambiente e sua sintaxe, com o propósito musical de criação a partir da formalização e controle de objetos musicais.

Para ser mais concreto, o ensino da EAC, no âmbito do "Cursus de composição e informática musical (sic)", no IRCAM, é realizado em duas etapas. O primeiro é o trabalho em classe, e o segundo é a realização de projetos musicais pessoais ${ }^{37}$.

No que diz respeito ao trabalho em sala de aula, as aulas são dadas na forma de uma oficina, combinando informações teóricas com a realização prática, baseadas em escuta, reflexão e integração pelo aluno dos conceitos e ferramentas apresentadas. Cada curso ocorre em uma jornada de 6 horas ( 2 x 3 horas). Após um conjunto de dias de curso (entre 3 e 4 dias), correspondentes a um assunto específico ${ }^{38}$, os alunos recebem a tarefa de "escrever" (calcular) um "estudo". Este estudo é, muitas vezes, uma sequência musical curta realizada no ambiente de computador usado (OpenMusic, no nosso caso). Se o assunto for técnico, proporemos, por exemplo, um "estudo de editor"; se o assunto for musical, proporemos um "estudo estocástico", um "estudo combinatório" ou um "estudo espectral".

O ensino dirigido aos jovens criadores é especializado, pois eles sempre têm a necessidade de aplicar o conhecimento imediatamente para compreendê-lo e integrá-lo totalmente - isto com o objetivo de testar sua estética e preocupações musicais no ambiente de EAC proposto.

\section{Conclusões}

A consciência dos pontos expostos nos permite sugerir que, sob os termos CAC ou $E A C$, se esconde uma sinédoque. Falar sobre CAC ou EAC diz respeito a um conjunto de disciplinas. Este ponto nos permite identificar alguns problemas que podem se tornar armadilhas conceituais.

A primeira dessas armadilhas, a mais comum, é reduzir a aprendizagem da EAC, ou de uma disciplina de música por computador, à aprendizagem de uma ferramenta. Isso equivale a reduzir o aprendizado da escrita à aprendizagem dos editores de texto, reduzir o aprendizado do piano à aprendizagem dos tipos de toques, reduzir a aprendizagem da escrita musical à aprendizagem dos programas de notação e, em última instância, reduzir a Astronomia ao estudo do telescópio. Em comparação com os "instrumentos musicais tradicionais" (piano, violino etc.), as ferramentas informáticas evoluem, aparecem e desaparecem em uma escala de tempo muito menor. Como basear um conheci- 
mento, uma disciplina em ferramentas tão efêmeras ${ }^{39}$ ?

A segunda armadilha é a de não ter uma visão crítica dos ambientes de computação e esquecer que são conjuntos de representações, que induzem modos de operação. Trabalhar em um ambiente de computador é manipular e pensar as representações.

A terceira armadilha é o fato de que, apesar dos ambientes transmitirem conhecimento, eles não são o próprio conhecimento. Este ponto é importante porque requer uma atualização constante do conhecimento auxiliar. No caso da EAC, isto se relaciona com as capacidades de formalização e as técnicas já desenvolvidas. Um exemplo clássico são as "bibliotecas externas". As bibliotecas externas OpenMusic, como "lz"40, "omalea"41, "Perfil"42, "Omchroma"43 ou "repmus"44, que requerem conhecimentos extras para serem usados. Em Max/MSP ou PureData o fenômeno é análogo. Os ambientes informáticos utilizados sem este conhecimento prévio tornam-se fontes de frustração. Conhecer apenas a ferramenta é insuficiente, assim como o simples conhecimento da teoria, sem que possa ser efetivada, também não é suficiente.

Para resumir, diremos que as principais questões de ensino (e de aprendizagem) da EAC estão situadas particularmente no nível da modelização e da formalização do pensamento, do conhecimento dos métodos de trabalho e da relação entre cálculo e música, culminando no desenvolvimento de um solfejo de modelos pelo compositor.

Finalmente, "podemos aprender OpenMusic em três dias"? Quando percebemos a importância dessas reflexões, isso realmente não faz mais sentido.

\section{Referências}

AGON, Carlos Augusto. OpenMusic: Un langage visuel pour la composition musicale assistée par ordinateur. Thèse (Doctorat) - Paris VIII, 1998.

AGON, Carlos Augusto; ANDREATTA, Moreno; ASSAYAG, Gérard; SCHAUB, Stéphan. Formal Aspects of lannis Xenakis' "Symbolic Music": A Computer- Aided Exploration of Compositional Processes. Journal of New Music Research, v. 33, n. 2, p. 145-159, june 2004.

AGON, Carlos Augusto; ASSAYAG, Gérard; BRESSON, Jean (Eds.). The om composer's book. V. 1. Sampzon: Sciences Editions Delatour France; IRCAM, 2006. Collection Mu-

\footnotetext{
39 Em discussão com Denis Lorain (2009), em St-Étienne, sobre o efêmero das ferramentas informáticas e a persistência do ensino, chegamos à conclusão de que, para um ensinamento da EAC, geral e tão duradouro quanto possível, ele deve usar uma linguagem de programação como um ambiente de computação.

40 Biblioteca de análise e geração de sequências com base no algoritmo lempelZiv (Gérard Assayag, Shlomo Dubnov).

41 Ferramentas de geração aleatória baseadas em distribuições de probabilidade (Mikhail Malt).

42 Biblioteca de manipulação de sequências melódicas, representadas como de curvas geométricas (Mikhail Malt \& Jacopo Babolin Schillingi).

43 Bibliotecas para o controle do sintetizador de software CSound (http://csound.sourceforge.net/), desenvolvido por Marco Stroppa e Jean Bresson.

44 Biblioteca de várias pesquisas (telas, trilhas em gráficos etc.) da equipe de Representação Musical IRCAM.
} 
sique.

BIDLACK, Richard. Chaotic Systems as Simple (but complex) Compositional Algorithms. Computer Music Journal, MIT-Press, v. 16, n. 3, Fall 1992.

BOULEZ, P.; GREUSSAY, P. Et la musique. Entretien avec Philippe Manoury. Traverses, n. 44-45 - Machines Virtuelles, Paris, Centre de Création Industrielle, Centre Georges Pompidou, p. 129-137, 1988.

BRESSON, Jean; AGON, Carlos Augusto; ASSAYAG, Gérard (Eds.). The om composer's book. V. 2. Sampzon: Sciences Editions Delatour France; IRCAM, 2008. Collection Musique.

HIRS, Rozalie; GILMORE, Bob (Eds.). Contemporary Compositional Techniques and OpenMusic. Musique/Sciences Series, IRCAM/Delatour, France, en collaboration avec la Amsterdam School of the Arts, Amsterdam, Netherlands, 2009.

LEVY, Pierre. La Machine Univers. Paris, France: Editions la Découverte, 1987

MALT, Mikhail. Lambda 3.99 (Chaos et Composition Musicale). Troisièmes Journées d'Informatique Musicale JIM 96, Ile de Tatihou, Normandie, France, 1996.

La composition assistée par ordinateur, modèles et calcul, quelques éléments de réflexion. In: POTTIER, Laurent (Ed.). Le calcul de la musique. Saint-Étienne: Publications de l'Université de Saint-Étienne, 2009. p. 163-224.

La représentation dans le cadre de la composition et de la musicologie assistées par ordinateur, mémoire d'Habilitation à Diriger des Recherches, Université de Strasbourg, soutenue le 04 février 2015. 332 p. Disponível em: <ftp://ftp.ircam.fr/private/pedagogie/mikhail/HDR_memoires/HDR_Le_tout_correct_0051.pdf>.

ROADS, Curtis. The Computer Music Tutorial. Cambridge: MIT Press, 1994.

VEITL, Anne. Musique serieuse et informatique: a formation musicale. en XIXe siècle -> 1983. 2008. p. 1-25. Disponível em: <www.tscimuse.org/biblios/veitl/chronologie19siecle- 1983.pdf>.

XENAKIS, Iannis. Musiques Formelles. Paris: Stock Musique, 1981. 